Der mIDentity mini bildet zukünftig das Einstiegsmodell der mlDentity Familie. Bei mIDentity Air und mIDentity Air+ wird weltweit erstmalig Bluetooth Technologie zur Übertragung der Transaktionsdaten vom Mobilgerät (iPhone, Tablet etc.) zu einer externen Hardware-Signaturkomponente genutzt. Die Bluetooth Technologie wird dabei durch zusätzliche Sicherheitsmaßnahmen den hohen Sicherheitsanforderungen der Übertragung per USB Kabel angepasst, bietet aber gleichzeitig auch die höchste Mobilität und Einfachheit der Benutzung durch das sogenannte "Just-Works"-Pairing. So können beispielsweise iPhone Benutzer auch die mIDentity-Technologie nutzen, da die komplette Übertragung der Daten über Bluetooth stattfindet. Das Modell „mIDentity Air+" verfügt sogar über ein eigenes 1,7" TFT-Farbdisplay zur vertrauenswürdigen Anzeige der Transaktionsdaten.

Erstmalig steht auch eine reine Softwarelösung für Apps zur Verfügung. Die „mIDentity App“ führt dazu spezifische Härtungsmaßnahmen ein, um auch Szenarien gerecht werden zu können, bei denen der Benutzer außer seinem Smartphone keine separate Hardware mit sich führen möchte oder kann. Die neue Aktivierungsprozedur, basierend auf KOBIL's Smart Signature Technology, ermöglicht eine Software-Signierung von Transaktionen, welche modular auf die Hardware Ausbaustufen mIDentity Air und Air+ erweitert werden kann. Somit bietet die mIDentity App auch eine kostengünstige Alternative zum SMS-TAN Verfahren, bei dem die Bank bzw. der Endkunde jede einzelne SMS bezahlen muss. Ein SDK wird erhältlich sein, um die neuen Sicherheitsfeatures in bestehende Banking Apps (Fat Clients) oder auch in Web-basierte Angebote (Web-Apps) sehr einfach und transparent zu integrieren.

Ergänzt werden die Produkte mit dem SSMS-Server, welcher eine einheitliche Verwaltung aller KOBIL-Produkte ermöglicht. Der SSMS-Server bildet das Herzstück der mIDentity Family, da alle Authentifikationen und Signierungen, dank der Dual Communication Technology über den SSMS-Server laufen und somit den Banken ein zweiter Kanal zur Verfügung gestellt wird, der nochmals getrennt gesichert ist. Dies ermöglicht maximale Sicherheit bei höchstem Bedienerkomfort.

Für weitere Informationen: http://www.kobil.com/de/produkte. html

\section{OpenVAS-5 mit neuen Funktionen}

Am 08.11.2011 hat das Bundesamt für Sicherheit in der Informationstechnik über die Verfügbarkeit einer neuen Generation von OpenVAS informiert.

Mit neuer Webseite und erweiterten Managementfunktionen spricht das Netzwerk-Sicherheitswerkzeug Open Vulnerability Assessment System (OpenVAS) weitere Nutzer an und geht mit großen Schritte auf die mittlerweile 5. Generation zu. OpenVAS-5 zeigt Veränderungen bei Wiederholungsprüfungen besser an. Neu sind zudem umfangreiche Filter- und Kategorisierungsmöglichkeiten und ein darauf basierender Frühwarnmechanismus. Die überarbeitete Webseite richtet sich stärker an neue Interessenten und ist erstmals in Deutsch verfügbar. Bundesbehörden, welche OpenVAS einsetzen möchten, stellt das BSI eine kostenfreie Bundeslizenz mit Unterstützung durch das Osnabrücker Unternehmen Greenbone Networks GmbH zur Verfügung.

In IT-Sicherheitskreisen ist OpenVAS international bekannt. Es kann als Freie Software (OSS) ohne Lizenzkosten beschafft und eingesetzt werden. Früher wurde OpenVAS vorrangig von Penetrationstestern explorativ verwendet. Inzwischen steht, auf diesen Sicherheitsscans aufbauend, vor allem die Verwendung von OpenVAS als Werkzeug für das Management von Sicherheitsrichtlinien und Schwachstellen von IT-Geräten mit Netzwerkanschluss im Vordergrund. Beim Einsatz können regelmäßig alle Geräte im Netz überprüft und die Prüfergebnisse übersichtlich erfasst und aufbereitet werden. Anhand von Schwachstellenbeschreibungen, beispielsweise den Common Vulnerabilities and Exposures (CVEs), können Sicherheitslücken schnell bewertet und Maßnahmen geplant werden.

OpenVAS bietet auch die Möglichkeit individuelle Sicherheitsrichtlinien zu erstellen. So hat das BSI mittlerweile über 100 als automatische Prüfungen implementierte IT-Grundschutz-Maßnahmen für OpenVAS verfügbar gemacht. Über 23000 weitere Prüfmodule sind erhältlich, deren Aktualisierungen automatisch nachgeladen werden. Dieser sogenannte „Feed Service" ist kryptographisch abgesichert.

Weitere Informationen zu OpenVAS auf der Website https:// www.bsi.bund.de/DE/Themen/ProdukteTools/OpenVAS/OpenVAS_ node.html

\section{Nano-SIM-Karte von G \& D}

Giesecke \& Devrient (G\&D) stellt mit der Nano-SIM die weltweit kleinste SIM-Karte vor. Die im Vergleich zur Micro-SIM um ein Drittel verkleinerte Karte ermöglicht Herstellern die Produktion leistungsstärkerer und schlankerer Geräte. Die Neuentwicklung von G\&D könnte bereits nächstes Jahr in den ersten mobilen Endgeräten eingesetzt werden.

Rund 20 Jahre nach der Produktion der international ersten SIMKarte wird damit der Trend zu kleineren Karten umgesetzt.

Im Vergleich zur Micro-SIM hat sich die Größe der Nano-SIM mit einem Format von zirka $12 \times 9 \mathrm{~mm}$ um etwa 30 Prozent verringert. Die Dicke der Karten hat sich um zirka 15 Prozent reduziert, was eine enorme technische Herausforderung darstellt. Der Größenunterschied zur heute am meist verbreiteten SIM-Karte beträgt sogar fast 60 Prozent. Endgeräteherstellern bringt die Nano-SIM den entscheidenden Vorteil, diesen Spielraum für andere Komponenten im Mobiltelefon nutzen zu können. So erlaubt die Nano-SIM beispielsweise den Einsatz größerer Speicher oder Akkus. Auch die Herstellung von schlankeren Gerätemodellen wird durch die deutlich kleineren und schmaleren Nano-SIM-Karten weiter vereinfacht.

Erste Samples wurden verschiedenen Mobilfunk-Netzbetreibern bereits zu Testzwecken zur Verfügung gestellt. Die Standardisierung der Nano-SIM wird voraussichtlich Ende dieses Jahres durch ETSI (European Telecommunications Standards Institute) erfolgen.

Für eine Rückwärtskompatibilität mit älteren Gerätemodellen sorgt eine Adapterlösung, durch die sich die Nano-SIM in alle gängigen mobilen Endgeräten integrieren und sich damit universell nutzen lässt.

\section{DGN PINtool: Signaturkarten einfach prüfen und verwalten}

Das Deutsche Gesundheitsnetz (DGN) hat für seine elektronischen Signaturkarten ein neues Werkzeug entwickelt, mit dem Anwender schnell und einfach die PINs ihrer Karte ändern können. Die ge- 